\title{
Xp22.31 Microdeletion due to \\ Microhomology-Mediated Break-Induced Replication in a Boy with Contiguous Gene Deletion Syndrome
}

\author{
Koki Nagai ${ }^{a}$ Hirohito Shimaa, c Miki Kamimura ${ }^{c}$ Junko Kanno ${ }^{c}$ Erina Suzukia \\ Akira Ishigurob $^{b}$ Satoshi Narumi ${ }^{a}$ Shigeo Kure ${ }^{c}$ Ikuma Fujiwarad \\ Maki Fukami ${ }^{a}$ \\ aDepartment of Molecular Endocrinology, National Research Institute for Child Health and Development, and \\ ${ }^{b}$ Department of Postgraduate Education and Training, National Center for Child Health and Development, Tokyo, \\ and ${ }^{\mathrm{C}}$ Department of Pediatrics, Tohoku University School of Medicine, and ${ }^{\mathrm{d}}$ Department of Pediatric Endocrinology \\ and Environmental Medicine, Tohoku University Graduate School of Medicine, Sendai, Japan
}

\section{Keywords}

ANOS1 - Copy number variation · Genome $\cdot \mathrm{MMBIR} \cdot \mathrm{STS}$.

$\mathrm{X}$ chromosome

\section{Abstract}

The Xp22.31 region is characterized by a low frequency of interspersed repeats and a low GC content. Submicroscopic deletions at Xp22.31 involving STS and ANOS1 (alias KAL1) underlie $\mathrm{X}$-linked ichthyosis and Kallmann syndrome, respectively. Of the known microdeletions at Xp22.31, a common approximately $1.5-\mathrm{Mb}$ deletion encompassing STS was ascribed to nonallelic homologous recombination, while 2 ANOS1-containing deletions were attributed to nonhomologous end-joining. However, the genomic bases of other microdeletions within the $\mathrm{Xp} 22.31$ region remain to be elucidated. Here, we identified a 2,735,696-bp deletion encompassing STS and ANOS1 in a boy with X-linked ichthyosis and Kallmann syndrome. The breakpoints of the deletion were located within Alu repeats and shared 2-bp microhomology. The fusion junction was not associated with nucleotide

\section{KARGER}

(C) 2017 S. Karger AG, Basel

E-Mail karger@karger.com

www.karger.com/cgr stretches, and the breakpoint-flanking regions harbored no palindromes or noncanonical DNA motifs. These results indicate that microhomology-mediated break-induced replication (MMBIR) can cause deletions at Xp22.31, resulting in contiguous gene deletion syndrome. It appears that interspersed repeats without other known rearrangement-inducing DNA features or high GC contents are sufficient to stimulate MMBIR at Xp22.31.

(c) 2017 S. Karger AG, Basel

The Xp22.31 region resides within a specific evolutionary stratum on the human $\mathrm{X}$ chromosome and is characterized by a low frequency of interspersed repeats and a low GC content [Ross et al., 2005]. This region contains several genes including STS, ANOS1 (alias KAL1), NLGN4X, HDHD1 (PUDP), PNPLA4, and the VCX cluster [Ross et al., 2005; Ben Khelifa et al., 2013]. Of these,

K.N., H.S., and M.K. contributed equally to this study.

Maki Fukami

National Research Institute for Child Health and Development

2-10-1 Okura, Setagaya

Tokyo 157-8535 (Japan)

E-Mail fukami-m@ncchd.go.jp 
STS is the causative gene for X-linked ichthyosis, while ANOS1 is associated with Kallmann syndrome characterized by anosmia and hypogonadotropic hypogonadism [Ballabio et al., 1987; Bick et al., 1992]. NLGN4X and the VCX genes have been implicated in intellectual development, although the functional significance of these genes remains under debate [Fukami et al., 2000; Jamain et al., 2003; Macarov et al., 2007; Mochel et al., 2008]. HDHD1 and PNPLA4 encode proteins that may be involved in nucleotide dephosphorylation and epidermal homeostasis, respectively [Gao and Simon, 2007; Preumont et al., 2010]; however, mutations in these genes have not been linked to human diseases. Various submicroscopic deletions within the Xp22.31 region have been identified in patients with X-linked ichthyosis and/or Kallmann syndrome, with or without intellectual disability [Martul et al., 1995; Maya-Núñez et al., 1998; Van Esch et al., 2005; Mochel et al., 2008]. In particular, an approximately $1.5-\mathrm{Mb}$ microdeletion encompassing STS represents a common cause of X-linked ichthyosis [Jimenez Vaca et al., 2001; Van Esch et al., 2005]. This deletion was attributed to nonallelic homologous recombination (NAHR) mediated by locus-specific low-copynumber repeats [Van Esch et al., 2005]. Conversely, NAHR is unlikely to be involved in other Xp22.31 microdeletions that are not associated with repetitive sequences. In 2008, Hershkovitz et al. characterized the genomic structures of 2 ANOS1-containing microdeletions harbored by members of the same family [Hershkovitz et al., 2008]. The breakpoints of these deletions shared no homology and had short nucleotide stretches at the fusion junctions, indicating that nonhomologous end-joining (NHEJ) underlies these copy number variations (CNVs) [Hershkovitz et al., 2008]. Nevertheless, there have been no further reports characterizing the breakpoints of CNVs at Xp22.31.

CNVs in the human genome are known to result from NAHR, NHEJ, microhomology-mediated break-induced replication (MMBIR), or fork stalling and template switching [Hastings et al., 2009]. Chromosomal instability leading to CNVs is determined by local genomic features such as repetitive sequences, high GC contents, and noncanonical (non-B) DNA motifs [Cooper et al., 2011]. Since the Xp22.31 region contains markedly fewer repeats and lower GC contents than the average of the human genome [Ross et al., 2005], the mechanism by which CNVs at Xp22.31 are created is of particular interest. In the present study, we identified and characterized a hitherto unreported submicroscopic deletion at Xp22.31.

\section{Patient and Methods}

\section{Patient}

A 6-month-old boy was referred to our clinic because of genital abnormalities and skin lesions. The boy was the first child of healthy nonconsanguineous Japanese parents. He was born at 38 weeks of gestation, with a birth weight of $3,078 \mathrm{~g}(+0.8 \mathrm{SD})$, a length of 49.0 $\mathrm{cm}(+0.4 \mathrm{SD})$, and a head circumference of $34.0 \mathrm{~cm}$ (+0.7 SD). Physical examinations at 6 months of age revealed a small penis (stretched penile length, $2.0 \mathrm{~cm}$; $-3.6 \mathrm{SD}$ ), bilateral undescended testes, and ichthyosis in the trunk and limbs. His height $(67.4 \mathrm{~cm} ;-0.2 \mathrm{SD})$ and weight $(7.2 \mathrm{~kg} ;-0.8 \mathrm{SD})$ were normal. His developmental milestones were normal, with head control at 4 months of age. Auditory tests yielded normal results. A gonadotropin-releasing hormone stimulation test showed a normal response of luteinizing hormone $[<0.07$ (baseline) and $2.5 \mathrm{mIU} / \mathrm{mL}$ (peak value); reference, $<0.4$ (baseline) and $0.4-6.0 \mathrm{mIU} / \mathrm{mL}$ (peak value)] and a hyper-response of folliclestimulating hormone [0.7 (baseline) and $24.7 \mathrm{mIU} / \mathrm{mL}$ (peak value); reference, 0.6-3.0 (baseline) and 6.3-15.6 $\mathrm{mIU} / \mathrm{mL}$ (peak value)]. Blood testosterone values were within the normal range $(<10 \mathrm{ng} /$ dL). G-banding showed a 46,XY karyotype. Brain magnetic resonance imaging detected olfactory sulcus and bulbs, but no olfactory tracts nor tubercles. Abdominal ultrasonograms and renal scintigrams showed right renal aplasia and left hydronephrosis.

\section{Molecular Analysis}

DNA samples were obtained from peripheral leukocytes of the patient. CNVs in the genome were examined by array-CGH using a catalog human array (SurePrint G3; $4 \times 180 \mathrm{~K}$ format, Agilent Technologies, Santa Clara, CA, USA). To characterize the breakpoint structure of a deletion, we amplified the genomic interval harboring the fusion junction. The sequences of primers were $5^{\prime}$-GAAACACGCCCAGTCTGATT- $3^{\prime}$ and $5^{\prime}$-GCCCAGCCCCACTTTATTTT- $3^{\prime}$. The PCR products were subjected to Sanger sequencing.

The size of the deletion was calculated from the data of the UCSC genome browser (https://genome.ucsc.edu/; hg19, build37). To clarify whether the breakpoints were associated with repeat sequences or known rearrangement-inducing DNA features, we analyzed a 150-bp region on either side of the breakpoints using Repeatmasker (http://www.repeatmasker.org), palindrome (http:// emboss.bioinformatics.nl/cgi-bin/emboss/palindrome), and non$\mathrm{B}$ database (https://nonb-abcc.ncifcrf.gov/). The non-B motifs analyzed were A-phased repeats, direct repeats, G-quadruplex motifs, inverted repeats, mirror repeats, short tandem repeats, and Z-DNA motifs [Cer et al., 2013].

\section{Results}

Array-CGH analysis identified a hemizygous approximately 2.7-Mb deletion within the Xp22.31 region (Fig. 1a). Breakpoint characterization revealed that the deletion was 2,735,696 bp in size (chrX:5,800,939$8,536,633)$ and contained all exons of NLGN4X, VCX3A, HDHD1, STS, VCX, PNPLA4, VCX2, and VCX3B, as well as the last 7 exons (exons 8-14) of ANOS1.
2
Cytogenet Genome Res 2017;151:1-4 DOI: $10.1159 / 000458469$
Nagai/Shima/Kamimura/Kanno/Suzuki/ Ishiguro/Narumi/Kure/Fujiwara/Fukami 
Fig. 1. Microdeletion identified in the boy. a Array-CGH analysis. The black, red, and green dots denote signals indicative of the normal, increased (higher than +0.4 ), and decreased (lower than -0.8) copy numbers, respectively. The black arrow indicates the deleted region. The genomic positions of the disease-associated genes and $A l u$ repeats at the breakpoints are shown. Chromosomal positions refer to those in the UCSC genome browser (hg19, build37). b Schematic representation of the breakpoints. The fusion junction is associated with a 2-bp overlap (yellow box). p-ter, short arm telomere; cen, centromere.

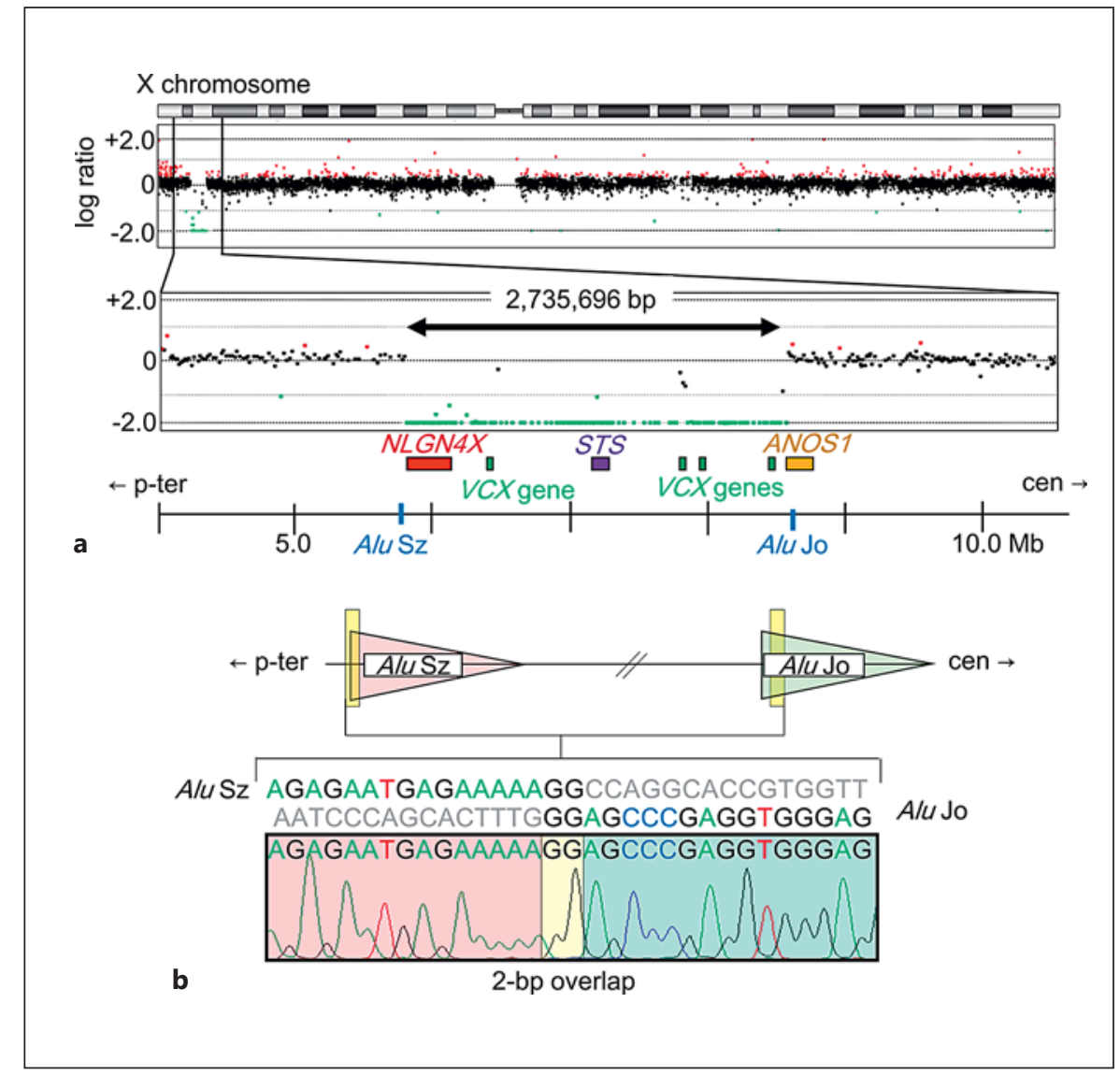

The telomeric and centromeric breakpoints were located at the boundary of Alu Sz (ChrX:5,800,937$5,801,248)$ and within Alu Jo (chrX:8,536,593-8,536,890), respectively (Fig. 1b). The 2 breakpoints shared no long homology but exhibited a microhomology of 2 bp (GG). No nucleotide stretches were observed at the fusion junction. The 150-bp breakpoint-flanking regions harbored no palindromes or non-B motifs.

\section{Discussion}

We identified a submicroscopic deletion within the Xp22.31 region in a boy with contiguous gene deletion syndrome. The breakpoints of the deletion exhibited a 2-bp microhomology, but were not accompanied by extensive homology or short nucleotide stretches at the fusion junction. This structure is indicative of MMBIR, which arises from replication fork collapse and subsequent microhomology-mediated template switching [Hastings et al., 2009]. Our results provide evidence that

MMBIR-Induced Xp22.31 Deletion replication-based errors can occur in genomic regions with sparse distribution of repeat sequences and GC nucleotides. Notably, the breakpoint-flanking regions of this deletion contained $A l u$ repeats but not palindromes or non-B motifs. In this regard, Hershkovitz et al. [2008] reported that the NHEJ-induced deletion in their cases was accompanied by $L 1$ repeats. Thus, MMBIR and NHEJ at Xp22.31 could be facilitated by repeat sequences independently of other rearrangement-inducing DNA features. It remains to be clarified whether the deletion in our patient is de novo or inherited from the phenotypically normal mother.

The clinical manifestations of our patient were consistent with hemizygous deletion of ANOS1 and STS. Specifically, the small penis and undescended testes can be ascribed to hypergonadotropic hypogonadism, and renal abnormalities have been observed in multiple patients with ANOS1 mutations [Söderlund et al., 2002; Laitinen et al., 2011]. Likewise, olfactory tract defects and ichthyosis are attributable to deficiencies of ANOS1 and STS, respectively [Ballabio et al., 1987; Söderlund et al., 2002; 
Laitinen et al., 2011]. While the patient exhibited no developmental delay despite lacking NLGN4X and the VCX genes, these results are consistent with prior observations that deletion of these genes is associated with a wide range of phenotypes including normal mental development [Macarov et al., 2007; Mochel et al., 2008]. However, considering the young age of the boy, follow-up studies are necessary to evaluate the clinical consequences of this deletion.

In summary, the results of this study show that MMBIR-mediated submicroscopic deletions at Xp22.31 underlie contiguous gene deletion syndrome. Interspersed repeats appear to be sufficient to stimulate MMBIR and NHEJ at Xp22.31 in the absence of other rearrangement-inducing DNA features or high GC contents.

\section{Acknowledgements}

This study was supported by Grants-in-Aid from the Japan Society for the Promotion of Science, and by Grants from the Ministry of Health, Labor and Welfare, the Japan Agency for Medical Research and Development, the National Center for Child Health and Development, and the Takeda foundation.

\section{Statement of Ethics}

This study was approved by the Institutional Review Board Committee at the National Center for Child Health and Development and performed after obtaining written informed consent.

\section{Disclosure Statement}

The authors have no competing interest to declare.

\section{References}

Ballabio A, Parenti G, Carrozzo R, Sebastio G, Andria $\mathrm{G}$, et al: Isolation and characterization of a steroid sulfatase cDNA clone: genomic deletions in patients with $\mathrm{X}$-chromosomelinked ichthyosis. Proc Natl Acad Sci USA 84: 4519-4523 (1987).

Ben Khelifa H, Soyah N, Ben-Abdallah-Bouhjar I, Gritly R, Sanlaville D, et al: Xp22.3 interstitial deletion: a recognizable chromosomal abnormality encompassing VCX3A and STS genes in a patient with X-linked ichthyosis and mental retardation. Gene 527:578-583 (2013).

Bick D, Franco B, Sherins RJ, Heye B, Pike L, Crawford J, et al: Intragenic deletion of the KALIG-1 gene in Kallmann's syndrome. N Engl J Med 326:1752-1755 (1992).

Cer RZ, Donohue DE, Mudunuri US, Temiz NA, Loss MA, et al: Non-B DB v2.0: a database of predicted non-B DNA-forming motifs and its associated tools. Nucleic Acids Res 41:D94100 (2013)

Cooper DN, Bacolla A, Férec C, Vasquez KM, Kehrer-Sawatzki H, Chen JM: On the sequence-directed nature of human gene mutation: the role of genomic architecture and the local DNA sequence environment in mediating gene mutations underlying human inherited disease. Hum Mutat 32:1075-1099 (2011).

Fukami M, Kirsch S, Schiller S, Richter A, Benes $\mathrm{V}$, et al: A member of a gene family on Xp22.3, $V C X-A$, is deleted in patients with $\mathrm{X}$-linked nonspecific mental retardation. Am J Hum Genet 67:563-573 (2000).
Gao JG, Simon M: A comparative study of human GS2, its paralogues, and its rat orthologue. Biochem Biophys Res Commun 360:501-506 (2007).

Hastings PJ, Lupski JR, Rosenberg SM, Ira G: Mechanisms of change in gene copy number. Nat Rev Genet 10:551-564 (2009).

Hershkovitz E, Loewenthal N, Peretz A, Parvari $\mathrm{R}$ : Testicular expressed genes are missing in familial X-linked Kallmann syndrome due to two large different deletions in daughter's $\mathrm{X}$ chromosomes. Horm Res 69:276-283 (2008).

Jamain S, Quach H, Betancur C, Råstam M, Colineaux $\mathrm{C}$, et al: Mutations of the X-linked genes encoding neuroligins NLGN3 and NLGN4 are associated with autism. Nat Genet 34:27-29 (2003).

Jimenez Vaca AL, Valdes-Flores Mdel R, RiveraVega MR, González-Huerta LM, KofmanAlfaro SH, Cuevas-Covarrubias SA: Deletion pattern of the STS gene in X-linked ichthyosis in a Mexican population. Mol Med 7:845-849 (2001).

Laitinen EM, Vaaralahti K, Tommiska J, Eklund E, Tervaniemi M, et al: Incidence, phenotypic features and molecular genetics of Kallmann syndrome in Finland. Orphanet J Rare Dis 6: 41 (2011).

Macarov M, Zeigler M, Newman JP, Strich D, Sury V, et al: Deletions of VCX-A and NLGN4: a variable phenotype including normal intellect. J Intellect Disabil Res 51:329-333 (2007).
Martul P, Pineda J, Levilliers J, Vazquez JA, Rodriguez-Soriano J, et al: Hypogonadotrophic hypogonadism with hyposmia, X-linked ichthyosis, and renal malformation syndrome. Clin Endocrinol (Oxf) 42:121-128 (1995).

Maya-Núñez G, Cuevas-Covarrubias S, Zenteno JC, Ulloa-Aguirre A, Kofman-Alfaro S, Méndez JP: Contiguous gene syndrome due to deletion of the first three exons of the Kallmann gene and complete deletion of the steroid sulphatase gene. Clin Endocrinol (Oxf) 48:713718 (1998)

Mochel F, Missirian C, Reynaud R, Moncla A: Normal intelligence and social interactions in a male patient despite the deletion of $N L$ GN4X and the VCX genes. Eur J Med Genet 51:68-73 (2008).

Preumont A, Rzem R, Vertommen D, Van Schaftingen $\mathrm{E}: H D H D 1$, which is often deleted in $\mathrm{X}$-linked ichthyosis, encodes a pseudouridine5'-phosphatase. Biochem J 431:237-244 (2010).

Ross MT, Grafham DV, Coffey AJ, Scherer S, McLay K, et al: The DNA sequence of the human X chromosome. Nature 434:325-337 (2005).

Söderlund D, Canto P, Méndez JP: Identification of three novel mutations in the KAL1 gene in patients with Kallmann syndrome. J Clin Endocrinol Metab 87:2589-2592 (2002).

Van Esch H, Hollanders K, Badisco L, Melotte C, Van Hummelen P, et al: Deletion of VCX-A due to NAHR plays a major role in the occurrence of mental retardation in patients with $\mathrm{X}$-linked ichthyosis. Hum Mol Genet 14: 1795-1803 (2005). Cytogenet Genome Res 2017;151:1-4
DOI: $10.1159 / 000458469$
Nagai/Shima/Kamimura/Kanno/Suzuki/ Ishiguro/Narumi/Kure/Fujiwara/Fukami 